\title{
The Tropical Totally Positive Grassmannian
}

\author{
DAVID SPEYER \\ Department of Mathematics, University of California, Berkeley \\ LAUREN WILLIAMS \\ Department of Mathematics, MIT, Cambridge \\ Received January 8, 2004; Revised February 17, 2005; Accepted February 17, 2005
}

\begin{abstract}
Tropical algebraic geometry is the geometry of the tropical semiring $(\mathbb{R}$, min, +$)$. The theory of total positivity is a natural generalization of the study of matrices with all minors positive. In this paper we introduce the totally positive part of the tropicalization of an arbitrary affine variety, an object which has the structure of a polyhedral fan. We then investigate the case of the Grassmannian, denoting the resulting fan $\operatorname{Trop}^{+} G r_{k, n}$. We show that $\operatorname{Trop}^{+} G r_{2, n}$ is the Stanley-Pitman fan, which is combinatorially the fan dual to the (type $A_{n-3}$ ) associahedron, and that $\operatorname{Trop}^{+} G r_{3,6}$ and Trop ${ }^{+} G r_{3,7}$ are closely related to the fans dual to the types $D_{4}$ and $E_{6}$ associahedra. These results are strikingly reminiscent of the results of Fomin and Zelevinsky, and Scott, who showed that the Grassmannian has a natural cluster algebra structure which is of types $A_{n-3}, D_{4}$, and $E_{6}$ for $G r_{2, n}, G r_{3,6}$, and $G r_{3,7}$. We suggest a general conjecture about the positive part of the tropicalization of a cluster algebra.
\end{abstract}

Keywords: tropical geometry, total positivity, cluster algebras, generalized associahedra, Grassmannian

\section{Introduction}

Tropical algebraic geometry is the geometry of the tropical semiring $(\mathbb{R}, \min ,+)$. Its objects are polyhedral cell complexes which behave like complex algebraic varieties. Although this is a very new field in which many basic questions have not yet been addressed (see [16] for a nice introduction), tropical geometry has already been shown to have remarkable applications to enumerative geometry (see [14]), as well as connections to representation theory (see $[2,3,12])$.

The classical theory of total positivity concerns matrices in which all minors are positive. However, in the past decade this theory has been extended by Lusztig (see $[10,11])$, who introduced the totally positive variety $G_{>0}$ in an arbitrary reductive group $G$ and the totally positive part $B_{>0}$ of a real flag variety $B$. In the process, Lusztig discovered surprising connections between his theory of canonical bases for quantum groups and the theory of total positivity.

In this paper we introduce the totally positive part (or positive part, for short) of the tropicalization of an arbitrary affine variety over the ring of Puiseux series, and then investigate what we get in the case of the Grassmannian $G r_{k, n}$. First we give a parameterization of the totally positive part of the Grassmannian, largely based on work of Postnikov [15], and then we compute its tropicalization, which we denote by $\operatorname{Trop}^{+} G r_{k, n}$. We identify $\operatorname{Trop}^{+} G r_{k, n}$ 
with a polyhedral subcomplex of the $\left(\begin{array}{l}n \\ k\end{array}\right)$-dimensional Gröbner fan of the ideal of Plücker relations, and then show that this fan, modulo its $n$-dimensional lineality space, is combinatorially equivalent to an $(n-k-1)(k-1)$-dimensional fan which we explicitly describe. As a special case, we show that Trop ${ }^{+} G r_{2, n}$ is a fan which appeared in the work of Stanley and Pitman (see [19]), which parameterizes certain binary trees, and which is combinatorially equivalent to the (type $A_{n}$ ) associahedron. We also show that $\operatorname{Trop}^{+} G r_{3,6}$ and $\operatorname{Trop}^{+} G r_{3,7}$ are fans which are closely related to the fans of the types $D_{4}$ and $E_{6}$ associahedra, which were first introduced in [5]. These results are strikingly reminiscent of the results of Fomin and Zelevinsky [3], and Scott [17], who showed that the Grassmannian has a natural cluster algebra structure which is of type $A_{n}$ for $G r_{2, n}$, type $D_{4}$ for $G r_{3,6}$, and type $E_{6}$ for $G r_{3,7}$. (Fomin and Zelevinsky proved the $G r_{2, n}$ case and stated the other results; Scott worked out the cluster algebra structure of all Grassmannians in detail.) Finally, we suggest a general conjecture about the positive part of the tropicalization of a cluster algebra.

\section{Definitions}

In this section we will define the tropicalization and positive part of the tropicalization of an arbitrary affine variety over the ring of Puiseux series. We will then describe the tropical varieties that will be of interest to us.

Let $\mathcal{C}=\bigcup_{n=1}^{\infty} \mathbb{C}\left(\left(t^{1 / n}\right)\right)$ and $\mathcal{R}=\bigcup_{n=1}^{\infty} \mathbb{R}\left(\left(t^{1 / n}\right)\right)$ be the fields of Puiseux series over $\mathbb{C}$ and $\mathbb{R}$. Every Puiseux series $x(t)$ has a unique lowest term $a t^{u}$ where $a \in \mathbb{C}^{*}$ and $u \in \mathbb{Q}$. Setting $\operatorname{val}(f)=u$, this defines the valuation map val $:\left(\mathcal{C}^{*}\right)^{n} \rightarrow \mathbb{Q}^{n},\left(x_{1}, \ldots, x_{n}\right) \mapsto$ $\left(\operatorname{val}\left(x_{1}\right), \ldots, \operatorname{val}\left(x_{n}\right)\right)$. We define $\mathcal{R}^{+}$to be $\{x(t) \in \mathcal{C} \mid$ the coefficient of the lowest term of $x(t)$ is real and positive . We will discuss the wisdom of this definition later; for practically all purposes, the reader may think of $\mathcal{C}$ as if it were $\mathbb{C}$ and of $\mathcal{R}^{+}$as if it were $\mathbb{R}^{+}$.

Let $I \subset \mathcal{C}\left[x_{1}, \ldots, x_{n}\right]$ be an ideal. We define the tropicalization of $V(I)$, denoted Trop $V(I)$, to be the closure of the image under val of $V(I) \cap\left(\mathcal{C}^{*}\right)^{n}$, where $V(I)$ is the variety of $I$. Similarly, we define the positive part of Trop $V(I)$, which we will denote as Trop $^{+} V(I)$, to be the closure of the image under val of $V(I) \cap\left(\mathcal{R}^{+}\right)^{n}$. Note that Trop $V$ and $\operatorname{Trop}^{+} V$ are slight abuses of notation; they depend on the affine space in which $V$ is embedded and not solely on the variety $V$.

If $f \in \mathcal{C}\left[x_{1}, \ldots, x_{n}\right] \backslash\{0\}$, let the initial form in $(f) \in \mathbb{C}\left[x_{1}, \ldots, x_{n}\right]$ be defined as follows: write $f=t^{a} g$ for $a \in \mathbb{Q}$ chosen as large as possible such that all powers of $t$ in $g$ are nonnegative. Then in $(f)$ is the polynomial obtained from $g$ by plugging in $t=0$. If $f=0$, we set in $(f)=0$. If $w=\left(w_{1}, \ldots, w_{n}\right) \in \mathbb{R}^{n}$ then $\operatorname{in}_{w}(f)$ is defined to be $\operatorname{in}\left(f\left(x_{i} t^{w_{i}}\right)\right)$. If $I \subset \mathcal{C}\left[x_{1}, \ldots, x_{n}\right]$ then $\operatorname{in}_{w}(I)$ is the ideal generated by $\operatorname{in}_{w}(f)$ for all $f \in I$. It was shown in [18] that Trop $V(I)$ consists of the collection of $w$ for which $\operatorname{in}_{w}(I)$ contains no monomials. The essence of this proof was the following:

Proposition 2.1 ([18]) If $w \in \mathbb{Q}^{n}$ and $\operatorname{in}_{w}(I)$ contains no monomial then $V\left(\operatorname{in}_{w}(I)\right) \cap\left(\mathbb{C}^{*}\right)^{n}$ is nonempty and any point $\left(a_{1}, \ldots, a_{n}\right)$ of this variety can be lifted to a point $\left(\tilde{a}_{1}, \ldots, \tilde{a}_{n}\right) \in$ $V(I)$ with the leading term of $\tilde{a}_{i}$ equal to $a_{i} t^{w_{i}}$.

We now prove a similar criterion to characterize the points in $\operatorname{Trop}^{+} V(I)$. 
Proposition 2.2 A point $w=\left(w_{1}, \ldots, w_{n}\right)$ lies in $\operatorname{Trop}^{+} V(I)$ if and only if $\operatorname{in}_{w}(I)$ does not contain any nonzero polynomials in $\mathbb{R}^{+}\left[x_{1}, \ldots, x_{n}\right]$.

In order to prove this proposition, we will need the following result of [13], which relies heavily on a result of [9].

Proposition 2.3 ( [13]) An ideal I of $\mathbb{R}\left[x_{1}, \ldots, x_{n}\right]$ contains a nonzero element of $\mathbb{R}^{+}$ $\left[x_{1}, \ldots, x_{n}\right]$ if and only if $\left(\mathbb{R}^{+}\right)^{n} \cap V\left(\operatorname{in}_{\eta}(I)\right)=\emptyset$ for all $\eta \in \mathbb{R}^{n}$.

We are now ready to prove Proposition 2.2.

Proof: Define $T \subset \mathbb{Q}^{n}$ to be the image of $V(I) \cap\left(\mathcal{R}^{+}\right)^{n}$ under val. Let $U$ denote the subset of $\mathbb{R}^{n}$ consisting of those $w$ for which $\operatorname{in}_{w}(I)$ contains no polynomials with all positive terms. By definition, Trop ${ }^{+} V(I)$ is the closure of $T$ in $\mathbb{R}^{n}$. We want to show that the closure of $T$ is $U$.

It is obvious that $T$ lies in $U$. The subset $U$ is closed, as the property that $\operatorname{in}_{w}(f)$ has only positive terms is open as $w$ varies. Thus, the closure of $T$ lies in $U$.

Conversely, suppose that $w \in U$. Then, by Proposition 2.3 , for some $\eta \in \mathbb{R}^{n},\left(\mathbb{R}^{+}\right)^{n} \cap$ $V\left(\operatorname{in}_{\eta}\left(\operatorname{in}_{w}(I)\right)\right) \neq \emptyset$. For $\epsilon>0$ sufficiently small, we have $\operatorname{in}_{\eta}\left(\operatorname{in}_{w}(I)\right)=\operatorname{in}_{\epsilon \eta+w}(I)$. Therefore we can find a sequence $w_{1}, w_{2}, \ldots$ approaching $w$ with $\left(\mathbb{R}^{+}\right)^{n} \cap V\left(\operatorname{in}_{w_{i}}(I)\right) \neq \emptyset$.

As $w$ varies, $\operatorname{in}_{w}(I)$ takes on only finitely many values, and the subsets of $\mathbb{R}^{n}$ on which $\operatorname{in}_{w}(I)$ takes a specific value form the relative interiors of the faces of a complete rational complex known as the Gröbner complex (see [21]). These complexes are actually fans when $I$ is defined over $\mathbb{R}$ ([20]). Therefore, we may perturb each $w_{i}$, while preserving $\operatorname{in}_{w_{i}}(I)$, in order to assume that the $w_{i} \in \mathbb{Q}^{n}$ and we still have $w_{i} \rightarrow w$. Then, by Proposition 2.1, each $w_{i} \in T$, so $w$ is in the closure of $T$ as desired.

Corollary 2.4 Trop $V(I)$ and $\operatorname{Trop}^{+} V(I)$ are closed subcomplexes of the Gröbner complex. In particular, they are polyhedral complexes. If I is defined over $\mathbb{R}$, then $\operatorname{Trop} V(I)$ and Trop $^{+} V(I)$ are closed subfans of the Gröbner fan.

One might wonder whether it would be better to modify the definition of $\mathcal{R}^{+}$to require that our power series lie in $\mathcal{R}$. This definition, for example, is more similar to the appearance of the ring of formal powers series in [12]. One can show that in the case of the Grassmanian, this difference is unimportant. Moreover, the definition used here has the advantage that it makes it easy to prove that the positive part of the tropicalization is a fan.

Suppose $V(I) \subset \mathcal{C}^{m}$ and $V(J) \subset \mathcal{C}^{n}$ are varieties and we have a rational map $f$ : $\mathcal{C}^{m} \rightarrow \mathcal{C}^{n}$ taking $V(I) \rightarrow V(J)$. Unfortunately, knowing val $\left(x_{i}\right)$ for $1 \leq i \leq m$ does not in general determine val $\left(f\left(x_{1}, \ldots, x_{m}\right)\right)$, so we don't get a nice map $\operatorname{Trop} V(I) \rightarrow \operatorname{Trop} V(J)$. However, suppose that $f$ takes the positive points of $V(I)$ surjectively onto the positive points of $V(J)$ and suppose that $f=\left(f_{1}, \ldots, f_{n}\right)$ is subtraction-free, that is, the formulas for the $f_{i}$ 's are rational functions in the $x_{i}$ 's whose numerators and denominators have positive coefficients. Define Trop $f: \mathbb{R}^{m} \rightarrow \mathbb{R}^{n}$ by replacing every $\times$ in $f$ with a + , every/with a - , every + with a min and every constant $a$ with $\operatorname{val}(a)$. 
Proposition 2.5 Suppose $V(I) \subset \mathcal{C}^{m}$ and $V(J) \subset \mathcal{C}^{n}$ are varieties. Let $f: \mathcal{C}^{m} \rightarrow \mathcal{C}^{n}$ be a subtraction-free rational map taking $V(I)$ to $V(J)$ such that $V(I) \cap\left(\mathcal{R}^{+}\right)^{m}$ surjects onto $V(J) \cap\left(\mathcal{R}^{+}\right)^{n}$. Then Trop $f$ takes $\operatorname{Trop}^{+} V(I)$ surjectively onto $\operatorname{Trop}^{+} V(J)$.

Proof: This follows immediately from the formulas val $(x+y)=\min (\operatorname{val}(x), \operatorname{val}(y))$ and $\operatorname{val}(x y)=\operatorname{val}(x)+\operatorname{val}(y)$ for $x$ and $y \in \mathcal{R}^{+}$.

We now define the objects that we will study in this paper. Fix $k$ and $n$, and let $N=\left(\begin{array}{l}n \\ k\end{array}\right)$. Fix a polynomial ring $S$ in $N$ variables with coefficients in a commutative ring. The Plücker ideal $I_{k, n}$ is the homogeneous prime ideal in $S$ consisting of the algebraic relations (called Plücker relations) among the $k \times k$ minors of any $k \times n$-matrix with entries in a commutative ring.

Classically, the Grassmannian $G r_{k, n}$ is the projective variety in $\mathbb{P}_{\mathbb{C}}^{N-1}$ defined by the ideal $I_{k, n}$ of Plücker relations. We write $G r_{k n}(\mathcal{C})$ for the variety in $\mathbb{P}_{\mathcal{C}}^{N-1}$ defined by the same equations. Similarly, we write $G r_{k, n}(\mathbb{R})$ for the real points of the Grassmannian, $G r_{k, n}\left(\mathbb{R}^{+}\right)$for the real positive points, $G r_{k, n}\left(\mathcal{R}^{+}\right)$for those points of $G r_{k, n}(\mathcal{C})$ all of whose coordinates lie in $\mathcal{R}^{+}$and so on. We write $G r_{k, n}(\mathbb{C})$ when we want to emphasize that we are using the field $\mathbb{C}$, and use $G r_{k, n}$ when discussing results that hold with no essential modification for any field. The totally positive Grassmannian is the set $G r_{k, n}\left(\mathbb{R}^{+}\right)$.

An element of $G r_{k, n}$ can be represented by a full rank $k \times n$ matrix $A$. If $K \in\left(\begin{array}{c}{[n]} \\ k\end{array}\right)$, where $[n]$ denotes the set $\{1, \ldots, n\}$, we define the Plücker coordinate $\Delta_{K}(A)$ to be the minor of $A$ corresponding to the columns of $A$ indexed by $K$. We identify the element of the Grassmannian with the matrix $A$ and with its set of Plücker coordinates (which satisfy the Plücker relations).

Our primary object of study is the tropical positive Grassmannian $\operatorname{Trop}^{+} G r_{k, n}$, which is a fan, by Corollary 2.4. As in [18], this fan has an $n$-dimensional lineality space. Let $\phi$ denote the map from $\left(\mathbb{C}^{*}\right)^{n}$ into $\left(\mathbb{C}^{*}\right)^{k}$ which sends $\left(a_{1}, \ldots, a_{n}\right)$ to the $\left(\begin{array}{l}n \\ k\end{array}\right)$-vector whose $\left(i_{1}, \ldots, i_{k}\right)$-coordinate is $a_{i_{1}} a_{i_{2}} \cdots a_{i_{k}}$. We abuse notation by also using $\phi$ for the same map $\left(\mathcal{C}^{*}\right)^{n} \rightarrow\left(\mathcal{C}^{*}\right)^{k}$. Let Trop $\phi$ denote the corresponding linear map which sends $\left(a_{1}, \ldots, a_{n}\right)$ to the $\left(\begin{array}{l}n \\ k\end{array}\right)$-vector whose $\left(i_{1}, \ldots, i_{k}\right)$-coordinate is $a_{i_{1}}+a_{i_{2}}+\cdots+a_{i_{k}}$. The map Trop $\phi$ is injective, and its image is the common lineality space of all cones in Trop $G r_{k, n}$.

\section{Parameterizing the totally positive Grassmannian $G r_{k, n}\left(\mathbb{R}^{+}\right)$}

In this section we explain two equivalent ways to parameterize $G r_{k, n}\left(\mathbb{R}^{+}\right)$, as well as a way to parameterize $G r_{k, n}\left(\mathbb{R}^{+}\right) / \phi\left(\left(\mathbb{R}^{+}\right)^{n}\right)$. The first method, due to Postnikov [15], uses a certain directed graph $\mathrm{Web}_{k, n}$ with variables associated to each of its $2 k(n-k)$ edges. The second method is closely related to the first and uses the same graph, but this time variables are associated to each of its $k(n-k)$ regions. This has the advantage of giving a bijection between $\left(\mathbb{R}^{+}\right)^{k(n-k)}$ and $G r_{k, n}\left(\mathbb{R}^{+}\right)$. Finally, we use Web $\mathrm{W}_{k, n}$ with variables labelling each of its $(k-1)(n-k-1)$ inner regions in order to give a bijective parameterization of $G r_{k, n}\left(\mathbb{R}^{+}\right) / \phi\left(\left(\mathbb{R}^{+}\right)^{n}\right)$.

Let $\mathrm{Web}_{k, n}$ be the directed graph which is obtained from a $k$ by $n-k$ grid, as shown in figure 1. It has $k$ incoming edges on the right and $n-k$ outgoing edges on the bottom, and 


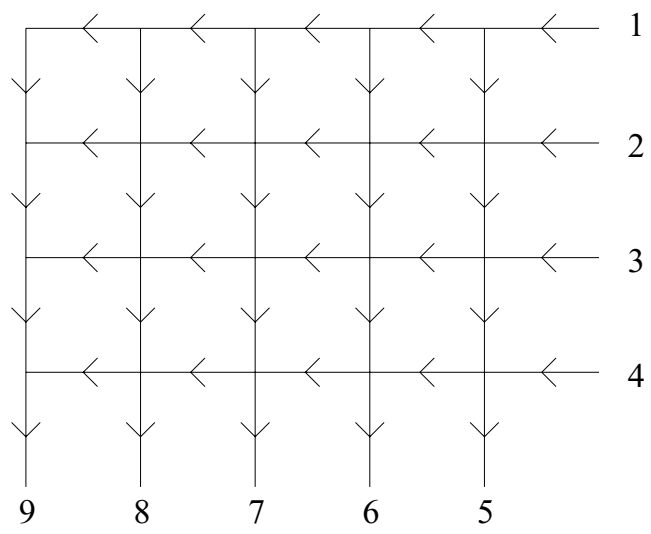

Figure 1. $\operatorname{Web}_{k, n}$ for $k=4$ and $n=9$.

the vertices attached to these edges are labelled clockwise from 1 to $n$. We denote the set of $2 k(n-k)$ edges by $E$. Let us associate a formal variable $x_{e}$ with each edge $e \in E$, and if there is no ambiguity, we abbreviate the collection $\left\{x_{e}\right\}$ by $x$. If $p$ is a path on $\mathrm{Web}_{k, n}$ (compatible with the directions of the edges), then we let $\operatorname{Prod}_{p}(x)$ denote $\prod_{e \in p} x_{e}$. And if $S$ is a set of paths on $\operatorname{Web}_{k, n}$, then we let $\operatorname{Prod}_{S}(x)$ denote $\prod_{p \in S} \operatorname{Prod}_{p}(x)$.

As in [15], we define a $k \times n$ matrix $A_{k, n}(x)$, whose entries $a_{i j}(x)$ are polynomials in the variables $x_{e}$, by the following equation:

$$
a_{i j}(x)=(-1)^{i+1} \sum_{p} \operatorname{Prod}_{p}(x)
$$

where the sum is over all directed paths $p$ from vertex $i$ to vertex $j$. Note that the $k \times k$ submatrix of $A_{k, n}(x)$ obtained by restricting to the first $k$ columns is the identity matrix. In particular, $A_{k, n}(x)$ is a full rank matrix and hence we can identify it with an element of $G r_{k, n}$. Also note that every element of the totally positive Grassmannian $G r_{k, n}\left(\mathbb{R}^{+}\right)$has a unique matrix representative whose leftmost $k \times k$ submatrix is the identity. We shall see that as the $\left\{x_{e}\right\}$ vary over $\left(\mathbb{R}^{+}\right)^{2 k(n-k)}$, the $A_{k, n}(x)$ range over all of $G r_{k, n}\left(\mathbb{R}^{+}\right)$.

We now show it is possible to express the maximal minors (Plücker coordinates) of $A_{k, n}(x)$ as subtraction-free rational expressions in the $x_{e}$, as shown in [15]. If $K \in\left(\begin{array}{c}{[n]} \\ k\end{array}\right)$, then let $\operatorname{Path}(K)$ denote the set

$\{S: S$ is a set of pairwise vertex-disjoint paths from $[k] \backslash(K \cap[k])$ to $K \backslash([k] \cap K)\}$.

Note that for $K=[k]$, we consider the empty set to be a legitimate set of pairwise vertexdisjoint paths.

Applied to $\mathrm{Web}_{k, n}$, Theorem 15.4 of [15] implies the following.

Proposition 3.1 The Plücker coordinates of $A_{k, n}(x)$ are given by

$$
\Delta_{K}\left(A_{k, n}(x)\right)=\sum_{S \in \operatorname{Path}(K)} \operatorname{Prod}_{S}(x) .
$$


Proof: We give a brief proof of this result: the main idea is to use the well-known GesselViennot trick [8]. First note that $a_{i j}(x)$ has a combinatorial interpretation: it is a generating function keeping track of paths from $i$ to $j$. Thus, the determinant of a $k \times k$ submatrix of $A_{k, n}(x)$ corresponding to the column set $K$ also has a combinatorial interpretation: it is a generating function for all sets of paths from $[k] \backslash(K \cap[k])$ to $K \backslash([k] \cap K)$, with the sign of each term keeping track of the number of crossings in the corresponding path set. What we need to show is that this is equal to the sum of the contributions from path sets which are pairwise vertex-disjoint. To see this, consider a path set which does have an intersection. Look at its lexicographically last intersection, and compare this path set to the one obtained from it by switching the two path tails starting at that point of intersection. These two path sets get different signs, but have equal weights, and hence they cancel each other out.

Proposition 3.1 allows us to define a map $\Phi_{0}:\left(\mathbb{R}^{+}\right)^{2 k(n-k)} \rightarrow G r_{k, n}\left(\mathbb{R}^{+}\right)$as follows. Let $K \in\left(\begin{array}{c}{[n]} \\ k\end{array}\right)$, and define $P_{K}:\left(\mathbb{R}^{+}\right)^{2 k(n-k)} \rightarrow \mathbb{R}^{+}$by

$$
P_{K}(x):=\sum_{S \in \operatorname{Path}(K)} \operatorname{Prod}_{S}(x)
$$

Clearly if we substitute positive values for each $x_{e}$, then $P_{K}(x)$ will be positive. We now define $\Phi_{0}$ by

$$
\Phi_{0}(x)=\left\{P_{K}(x)\right\}_{K \in\left(\begin{array}{c}
{[n]} \\
k
\end{array}\right)} \text {. }
$$

In other words, $\Phi_{0}$ is the map which sends a collection of positive real numbers $\left\{x_{e}\right\}$ to the element of $G r_{k, n}$ with Plücker coordinates $P_{K}(x)$ (which is identified with the matrix $\left.A_{k, n}(x)\right)$.

By Theorem 19.1 of [15], the map $\Phi_{0}$ is actually surjective: any point in $G r_{k, n}\left(\mathbb{R}^{+}\right)$can be represented as $A_{k, n}(x)$ for some positive choices of $\left\{x_{e}\right\}$. In summary, we have the following result (which will also be a consequence of our Theorem 3.3).

Proposition 3.2 The map $\Phi_{0}:\left(\mathbb{R}^{+}\right)^{2 k(n-k)} \rightarrow G r_{k, n}(\mathbb{R})$ is a surjection onto $G r_{k, n}\left(\mathbb{R}^{+}\right)$.

Unfortunately, the method we have just described uses $2 k(n-k)$ variables to parameterize a space of dimension $k(n-k)$. We will now explain how to do a substitution of variables which will reduce the number of variables to $k(n-k)$.

We define an inner region of $\mathrm{Web}_{k, n}$ to be a bounded component of the complement of $\mathrm{Web}_{k, n}$ (viewed as a subset of $\mathbb{R}^{2}$ ). And we define an outer region of $\mathrm{Web}_{k, n}$ to be one of the extra inner regions we would obtain if we were to connect vertices $i$ and $i+1$ by a straight line, for $i$ from 1 to $n-1$. A region is an inner or outer region. Note that there are $k(n-k)$ regions, which we denote by $R$, and there are $(k-1)(n-k-1)$ inner regions.

Let us label each region $r \in R$ with a new variable $x_{r}$, which we define to be the product of its counterclockwise edge variables divided by the product of its clockwise edge variables.

For example, the new variables $A, B, C$ shown in figure 2 would be defined by

$$
A:=\frac{x_{1} x_{2}}{x_{3} x_{4}}, \quad B:=\frac{x_{5} x_{6}}{x_{7}}, \quad C:=x_{8} x_{9} .
$$




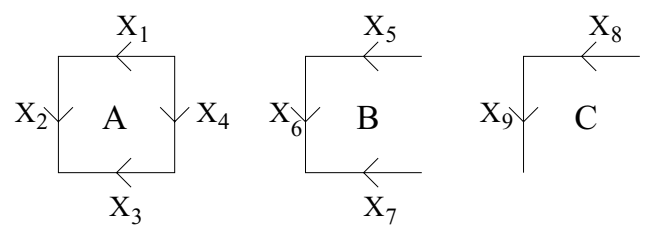

Figure 2. Substitution of variables.

It is easy to check that for a path $p$ on $\operatorname{Web}_{k, n}, \operatorname{Prod}_{p}(x)$ is equal to the product of the variables attached to all regions below $p$. Since $\operatorname{Prod}_{S}(x)$ and $A_{k, n}(x)$ were defined in terms of the $\operatorname{Prod}_{p}(x)$ 's, we can redefine these expressions in terms of the $k(n-k)$ region variables. Proposition 3.2 still holds, but our map is now a map $\Phi_{1}$ from $\left(\mathbb{R}^{+}\right)^{k(n-k)}$ onto $G r_{k, n}\left(\mathbb{R}^{+}\right)$, taking the region variables $\left\{x_{r}\right\}$ to the element of $G r_{k, n}\left(\mathbb{R}^{+}\right)$represented by $A_{k, n}(x)$.

Since we are now parameterizing a space of dimension $k(n-k)$ with $k(n-k)$ variables, we should have a bijection. We shall prove that this is so by constructing the inverse map.

Theorem 3.3 The map $\Phi_{1}:\left(\mathbb{R}^{+}\right)^{k(n-k)} \rightarrow G r_{k, n}\left(\mathbb{R}^{+}\right)$, which maps $\left\{x_{r}\right\}_{r \in R}$ to the Grassmannian element represented by $A_{k, n}(x)$, is a bijection.

Before we prove this theorem, we need a lemma about matrices and their minors. We use a very slight generalization of a lemma which appeared in [4]. For completeness, we include the proof of this lemma. First we must define some terminology. Let $M$ be a $k \times n$ matrix. Let $\Delta_{I, J}$ denote the minor of $M$ which uses row set $I$ and column set $J$. We say that $\Delta_{I, J}$ is solid if $I$ and $J$ consist of several consecutive indices; if furthermore $I \cup J$ contains 1 , we say that $\Delta_{I, J}$ is initial. Thus, an initial minor is a solid minor which includes either the first column or the first row.

Lemma 3.4 ([4]) A matrix $M$ is uniquely determined by its initial minors provided that all these minors are nonzero.

Proof: Let us show that each matrix entry $x_{i j}$ of $M$ is uniquely determined by the initial minors. If $i=1$ or $j=1$, there is nothing to prove, since $x_{i j}$ is an initial minor. Assume that $\min (i, j)>1$. Let $\Delta$ be the initial minor whose last row is $i$ and last column is $j$, and let $\Delta^{\prime}$ be the initial minor obtained from $\Delta$ by deleting this row and column. Then $\Delta=\Delta^{\prime} x_{i j}+P$, where $P$ is a polynomial in the matrix entries $x_{i^{\prime} j^{\prime}}$ with $\left(i^{\prime}, j^{\prime}\right) \neq(i, j)$ and $i^{\prime} \leq i, j^{\prime} \leq j$. Using induction on $i+j$, we can assume that each $x_{i^{\prime} j^{\prime}}$ that occurs in $P$ is uniquely determined by the initial minors, so the same is true of $x_{i j}=(\Delta-P) / \Delta^{\prime}$.

We now define a reflected initial minor to be a solid minor $\Delta_{I, J}$ such that $I$ contains $k$ or $J$ contains 1 . Thus, a reflected initial minor is a solid minor which includes either the first column or the last row. A trivial corollary of Lemma 3.4 is the following. 
Corollary 3.5 A matrix $M$ is uniquely determined by its reflected initial minors provided that all these minors are nonzero.

Now we are ready to prove Theorem 3.3.

Proof: To prove the theorem, we will construct an explicit inverse map $\Psi: G r_{k, n}\left(\mathbb{R}^{+}\right) \rightarrow$ $\left(\mathbb{R}^{+}\right)^{k(n-k)}$. The first step is to prove that $\Psi \Phi_{1}=\mathrm{id}$.

Let us index the regions in $\mathrm{Web}_{k, n}$ by ordered pairs $(i, j)$ as follows. Given a region, we choose $i$ to be the label of the horizontal wire which forms the upper boundary of the region, and choose $j$ to be the label of the vertical wire which forms the left boundary of the region. Now we define a map $K$ from the set of regions to $\left(\begin{array}{c}{[n]} \\ k\end{array}\right)$ by

$$
K(i, j):=\{1,2, \ldots, i-1\} \cup\{i+j-k, i+j-k+1, \ldots, j-1, j\} .
$$

If $(i, j)$ is not a region of $\mathrm{Web}_{k, n}$, then we define $K(i, j):=\emptyset$.

Let $A$ be a $k \times n$ matrix whose initial $k \times k$ minor is the identity. We define $\Psi(A)$ by

$$
(\Psi(A))_{(i, j)}:=\frac{\Delta_{K(i, j)}(A) \Delta_{K(i+1, j-2)}(A) \Delta_{K(i+2, j-1)}(A)}{\Delta_{K(i, j-1)}(A) \Delta_{K(i+1, j)}(A) \Delta_{K(i+2, j-2)}(A)} .
$$

Note that by convention, we define $\Delta_{\emptyset}$ to be 1 .

See figure 3 for the definition of $\Psi$ in the case of $G r_{3,6}\left(\mathbb{R}^{+}\right)$. Note that for brevity, we have omitted the $A$ 's from each term.

We claim that if $A=\Phi_{1} x$, then $\Psi \Phi_{1} x=x$. To prove this, we note that the variable in region $(i, j)$ can be expressed in terms of vertex-disjoint paths as follows.

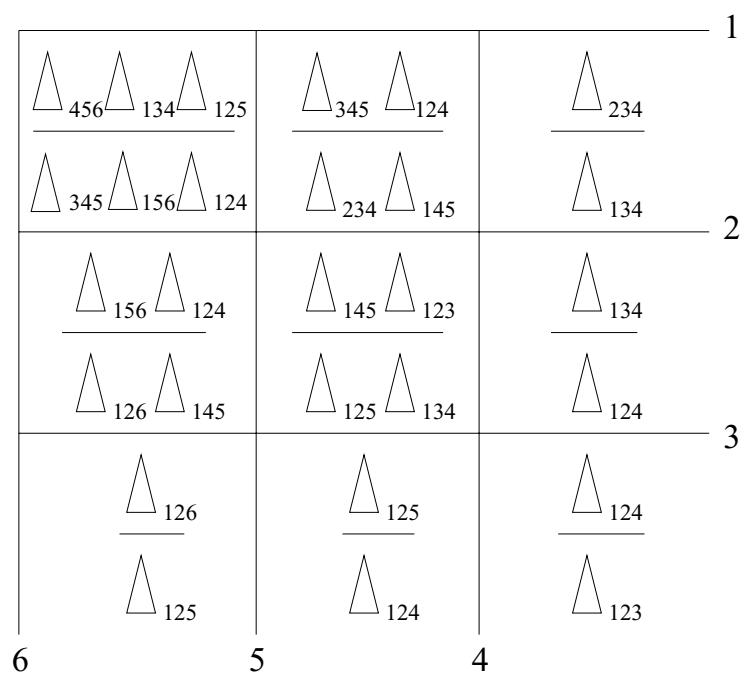

Figure 3. $\mathrm{Web}_{3,6}$. 
First observe that if $K(i, j) \neq \emptyset$ then there is a unique set of pairwise vertex-disjoint paths from $[k] \backslash([k] \cap K(i, j))$ to $K(i, j) \backslash([k] \cap K(i, j))$. If one examines the terms in (1) and draws in the six sets of pairwise vertex-disjoint paths on $\mathrm{Web}_{k, n}$ (say the three from the numerator in red and the three from the denominator in blue) then it is clear that every region in $\mathrm{Web}_{k, n}$ lies underneath an equal number of red and blue paths-except the region $(i, j)$, which lies underneath only one red path. Thus, by definition of the maps $P_{K}$, it follows that

$$
\left(\Psi \Phi_{1}(x)\right)_{(i, j)}=\frac{P_{K(i, j)}(x) P_{K(i+1, j-2)}(x) P_{K(i+2, j-1)}(x)}{P_{K(i, j-1)}(x) P_{K(i+1, j)}(x) P_{K(i+2, j-2)}(x)}=x_{(i, j)} .
$$

To complete the proof, it remains to show that $\Psi$ is injective. This will complete the proof because we know that $\Psi \Phi_{1} \Psi=\Psi$, and $\Psi$ injective then implies that $\Phi_{1} \Psi=$ id.

Choose an element of $G r_{k, n}\left(\mathbb{R}^{+}\right)$, which we identify with its unique matrix representative $A$ whose leftmost $k \times k$ minor is the identity. Let $\mathcal{V}$ denote the set of rational expressions which appear in the right-hand side of (1) for all regions $(i, j)$ in $\mathrm{Web}_{k, n}$. Let $\mathcal{P}$ denote the set of all individual Plücker coordinates which appear in $\mathcal{V}$. We prove that $\Psi$ is injective in two steps. First we show that the values of the expressions in $\mathcal{V}$ uniquely determine the values of the Plücker coordinates in $\mathcal{P}$. Next we show that the values of the Plücker coordinates in $\mathcal{P}$ uniquely determine the matrix $A$.

The first step is clear by inspection. We illustrate the proof in the case of $G r_{3,6}\left(\mathbb{R}^{+}\right)$. By the choice of $A, \Delta_{123}=1$. Looking at the rational expressions in figure 3 , we see that knowing the value $\frac{\Delta_{124}}{\Delta_{123}}$ determines $\Delta_{124}$; the value $\Delta_{124}$ together with the value $\frac{\Delta_{134}}{\Delta_{124}}$ determines $\Delta_{134}$; and similarly for $\Delta_{234}, \Delta_{125}, \Delta_{126}$. Next, these values together with the value $\frac{\Delta_{145} \Delta_{123}}{\Delta_{125} \Delta_{134}}$ determines $\Delta_{145}$, and so on.

For the second step of the proof, let $A^{\prime}$ denote the $k \times(n-k)$ matrix obtained from $A$ by removing the leftmost $k \times k$ identity matrix. Note that the values of the Plücker coordinates $\Delta_{K(i, j)}(A)$ (which are all elements of $\mathcal{P}$ ) determine the values of all of the reflected initial minors of $A^{\prime}$. (Each such Plücker coordinate is equal to one of the reflected initial minors, up to sign.) Thus, by Corollary 3.5, they uniquely determine the matrix $A^{\prime}$ and hence $A$. This completes the proof of Theorem 3.3.

Now let us parameterize $G r_{k, n}\left(\mathbb{R}^{+}\right) / \phi\left(\left(\mathbb{R}^{+}\right)^{n}\right)$. We shall show that we can do this by using variables corresponding to only the $(k-1)(n-k-1)$ inner regions of $\mathrm{Web}_{k, n}$.

First recall that the $n$-dimensional torus acts on $G r_{k, n}\left(\mathbb{R}^{+}\right)$by scaling columns of a matrix representative for $A \in G r_{k, n}\left(\mathbb{R}^{+}\right)$. (Although the torus has dimension $n$, this is actually just an $(n-1)$-dimensional action as the scalars act trivially.) Namely,

$$
\left(\lambda_{1}, \ldots, \lambda_{n}\right)\left(\begin{array}{lll}
a_{11} & \ldots & a_{1 n} \\
\vdots & & \vdots \\
a_{k 1} & \ldots & a_{k n}
\end{array}\right):=\left(\begin{array}{lll}
\lambda_{1} a_{11} & \ldots & \lambda_{n} a_{1 n} \\
\vdots & & \vdots \\
\lambda_{1} a_{k 1} & \ldots & \lambda_{n} a_{k n}
\end{array}\right)
$$

If $A \in G r_{k, n}\left(\mathbb{R}^{+}\right)$then we let $\bar{A}$ denote the torus orbit of $A$ under this action. Note that if $K=\left\{i_{1}, \ldots, i_{k}\right\}$, then $\Delta_{K}(\lambda A)=\lambda_{i_{1}} \lambda_{i_{2}} \ldots \lambda_{i_{k}} \Delta_{K}(A)$. 
We will now determine the corresponding torus action on $\left(\mathbb{R}^{+}\right)^{k(n-k)}$ such that the above bijection commutes with the actions. If $r$ is an internal region then $x_{r}$ is a ratio of Plücker coordinates with the same indices appearing on the top and bottom, so $x_{r}$ is not modified by the torus action. A simple computation shows that the torus acts transitively on the values of the outer region variables. Thus, taking the quotient by $\phi\left(\left(\mathbb{R}^{+}\right)^{n}\right)$ on the right hand side of the equation corresponds to forgetting the outer variables on the left.

Define a map $\Phi_{2}:\left(\mathbb{R}^{+}\right)^{(k-1)(n-k-1)} \rightarrow G r_{k, n}\left(\mathbb{R}^{+}\right) / \phi\left(\left(\mathbb{R}^{+}\right)^{n}\right)$ by lifting a point $c \in$ $\left(\mathbb{R}^{+}\right)^{(k-1)(n-k-1)}$ to any arbitrarily chosen point $\tilde{c} \in\left(\mathbb{R}^{+}\right)^{k(n-k)}$ and then mapping $c$ to $\overline{\Phi_{1}(\tilde{c})}$. We have just proven:

Theorem 3.6 The map $\Phi_{2}:\left(\mathbb{R}^{+}\right)^{(k-1)(n-k-1)} \rightarrow G r_{k, n}\left(\mathbb{R}^{+}\right) / \phi\left(\left(\mathbb{R}^{+}\right)^{n}\right)$ is a bijection.

\section{A fan associated to the tropical positive Grassmannian}

In this section we will construct a lower-dimensional fan associated to the tropical positive Grassmannian Trop ${ }^{+} G r_{k, n}$. By methods precisely analogous to those above, we can prove an analogue of Theorem 3.6 for the field of Puiseux series.

Theorem 4.1 The map $\Phi_{2}:\left(\mathcal{R}^{+}\right)^{(k-1)(n-k-1)} \rightarrow G r_{k, n}\left(\mathcal{R}^{+}\right) / \phi\left(\left(\mathcal{R}^{+}\right)^{n}\right)$ is a bijection.

This theorem allows us to compute $\operatorname{Trop}^{+} G r_{k, n} /(\operatorname{Trop} \phi)\left(\mathbb{R}^{n}\right)$ by applying the valuation map to the image of $\Phi_{2}$. By Proposition 2.5 we can tropicalize the map $\Phi_{2}$, obtaining the following surjective map.

$$
\operatorname{Trop} \Phi_{2}: \mathbb{R}^{(k-1)(n-k-1)} \rightarrow \operatorname{Trop}^{+} G r_{k, n} /(\operatorname{Trop} \phi)\left(\mathbb{R}^{n}\right)
$$

The map Trop $\Phi_{2}$ is the map we get by replacing multiplication with addition and addition with minimum in the definition of $\Phi_{2}$. Explicitly, it is defined as follows. Let $K \in\left(\begin{array}{c}{[n]} \\ k\end{array}\right)$, and let inner region variables take on values $\left\{x_{r}\right\}$ in $\mathbb{R}$. Outer region variables are chosen arbitrarily. If $p$ is a path on $\operatorname{Web}_{k, n}$ then we let $\operatorname{Sum}_{p}(x)$ denote the sum of all variables which label regions below $p$. Similarly, if $S$ is a set of paths, then let $\operatorname{Sum}_{S}(x)$ denote $\sum_{p \in S} \operatorname{Sum}_{p}(x)$. Now define $\operatorname{Trop} P_{K}(x): \mathbb{R}^{(k-1)(n-k-1)} \rightarrow \mathbb{R}$ by

$$
\operatorname{Trop} P_{K}(x):=\min \left\{\operatorname{Sum}_{S}(x): S \in \operatorname{Path}(K)\right\} .
$$

The map $\operatorname{Trop} \Phi_{2}$ is the map

$$
\operatorname{Trop} \Phi_{2}: \mathbb{R}^{(k-1)(n-k-1)} \rightarrow \operatorname{Trop}^{+} G r_{k, n} /(\operatorname{Trop} \phi)\left(\mathbb{R}^{n}\right) \subset \mathbb{R}^{N} /(\operatorname{Trop} \phi)\left(\mathbb{R}^{n}\right)
$$

given by

$$
\left(\operatorname{Trop} \Phi_{2}(x)\right)_{K}=P_{K}(x)
$$


Definition 4.2 The fan $F_{k, n}$ is the complete fan in $\mathbb{R}^{(k-1)(n-k-1)}$ whose maximal cones are the domains of linearity of the piecewise linear map Trop $\Phi_{2}$.

Because Trop $\Phi_{2}$ surjects onto $\operatorname{Trop}^{+} G r_{k, n} /(\operatorname{Trop} \phi)\left(\mathbb{R}^{n}\right)$, the fan $F_{k, n}$ reflects the combinatorial structure of the fan $\operatorname{Trop}^{+} G r_{k, n} /(\operatorname{Trop} \phi)\left(\mathbb{R}^{n}\right)$, which differs from Trop ${ }^{+} G r_{k, n}$ only through modding out by the linearity space. However, $F_{k, n}$ is much easier to work with, as it lives in $(k-1)(n-k-1)$-dimensional space as opposed to $\left(\begin{array}{l}n \\ k\end{array}\right)$-dimensional space.

Since the maps Trop $P_{K}$ are piecewise linear functions, to each one we can associate a fan $F\left(P_{K}\right)$ whose maximal cones are the domains of linearity for Trop $P_{K}$. It is clear that the fan $F_{k, n}$ is the simultaneous refinement of all of the fans $F\left(P_{K}\right)$.

From now on, by abuse of notation, we will refer to $\operatorname{Trop}^{+} G r_{k, n} /(\operatorname{Trop} \phi)\left(\mathbb{R}^{n}\right)$ as $\operatorname{Trop}^{+}$ $G r_{k, n}$.

\section{5. $\operatorname{Trop}^{+} G r_{2, n}$ and the associahedron}

In this section we will describe the fan $F_{2, n}$ associated to Trop ${ }^{+} G r_{2, n}$. We show that this fan is exactly the Stanley-Pitman fan $F_{n-3}$, which appeared in the work of Stanley and Pitman in [19]. In particular, the face poset of $F_{2, n}$, with a top element $\hat{1}$ adjoined, is isomorphic to the face lattice of the normal fan of the associahedron, a polytope whose vertices correspond to triangulations of the convex $n$-gon. (In the language of [1], this is the associahedron of type $A_{n-3}$.)

Let us first do the example of $\operatorname{Trop}^{+} \mathrm{Gr}_{2,5}$.

We use the web diagram $\mathrm{Web}_{2,5}$, as shown in figure 4. The maps Trop $P_{K}$ are given by:

$$
\begin{aligned}
& \operatorname{Trop} P_{1 j}=0 \text { for all } j \\
& \operatorname{Trop} P_{23}=0 \\
& \operatorname{Trop} P_{24}=\min \left(x_{1}, 0\right) \\
& \operatorname{Trop} P_{25}=\min \left(x_{1}+x_{2}, x_{1}, 0\right)
\end{aligned}
$$

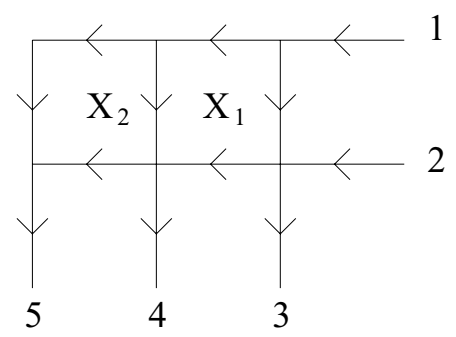

Figure 4. $\mathrm{Web}_{2,5}$. 


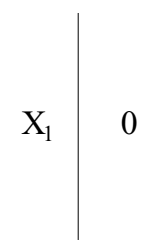

$\min \left(0, X_{1}\right)$

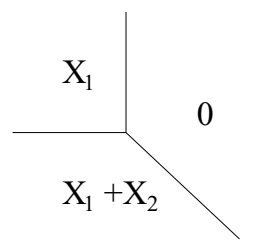

$\min \left(0, X_{1}, X_{1}+X_{2}\right)$

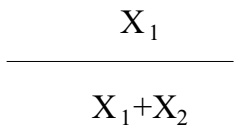

$\min \left(\mathrm{X}_{1}, \mathrm{X}_{1}+\mathrm{X}_{2}\right)$

Figure 5. Fans for Trop $P_{J}$.

$$
\begin{aligned}
& \operatorname{Trop} P_{34}=x_{1} \\
& \operatorname{Trop} P_{35}=\min \left(x_{1}, x_{1}+x_{2}\right) \\
& \operatorname{Trop} P_{45}=x_{1}+x_{2}
\end{aligned}
$$

Each map Trop $P_{K}: \mathbb{R}^{2} \rightarrow \mathbb{R}$ is piecewise linear and so gives rise to the complete fan $F\left(P_{K}\right)$. For example, the map $\operatorname{Trop} P_{24}$ is linear on the region $\left\{\left(x_{1}, x_{2}\right): x_{1} \geq 0\right\}$, where it is the function $\left(x_{1}, x_{2}\right) \mapsto 0$, and on the region $\left\{\left(x_{1}, x_{2}\right): x_{1} \leq 0\right\}$, where it is the function $\left(x_{1}, x_{2}\right) \mapsto x_{1}$. Thus, $F\left(P_{24}\right)$ is simply the subdivision of the real plane into the regions $x_{1} \geq 0$ and $x_{1} \leq 0$. The three nontrivial fans that we get from the maps Trop $P_{J}$ are shown in figure 5. In each picture, the maximal cones of each fan are separated by solid lines. $F_{2,5}$, which is the simultaneous refinement of the three nontrivial fans, is shown in figure 6.

In [18], it was shown that maximal cones of the fan Trop $G r_{2, n}$ correspond to trivalent trees on $n$ labelled leaves. It turns out that maximal cones of the fan Trop $G r_{2, n}^{+}$correspond to trivalent planar trees on $n$ labelled leaves, as is illustrated in figure 6 .

We will now describe the fan that appeared in [19], but first, we must review some notions about trees. A plane binary tree is a rooted tree such that each vertex has either two children designated as left and right, or none at all; and an internal vertex of a binary tree is a vertex which is not a leaf. A trivalent planar tree is an (unrooted) tree such that every vertex has degree three, and such that the leaves are labelled in a clockwise fashion. It is known that both plane binary trees with $n-1$ leaves, and trivalent planar trees with $n$ labelled leaves, are counted by the Catalan number $c_{n-2}=\frac{1}{n-1}\left(\begin{array}{c}2(n-2) \\ n-2\end{array}\right)$.

There is a simple bijection between such trivalent planar trees and plane binary trees: if $\mathrm{T}$ a trivalent planar tree, then simply contract the edge whose leaf is labelled 1 , and make this the root. This bijection is illustrated in figure 6 .

Let us now define the Stanley-Pitman fan $F_{n-3}$ in $\mathbb{R}^{n-3}$. (Note that we use different indices than are used in [19]). The maximal cones of $F_{n-3}$ are indexed by plane binary trees with $n-1$ leaves, in the following manner. Let $T$ be a plane binary tree with $n-1$ leaves. Label the internal vertices of $T$ with the numbers $1,2, \ldots n-2$ in the order of the first time we drop down to them from a child when doing a depth-first search from left to right starting at the root. (See figure 6 for examples.) Let $x_{1}, \ldots, x_{n-3}$ denote the coordinates in $\mathbb{R}^{n-3}$. If the internal vertex $i$ of $T$ is the parent of vertex $j$, and $i<j$, then associate with 


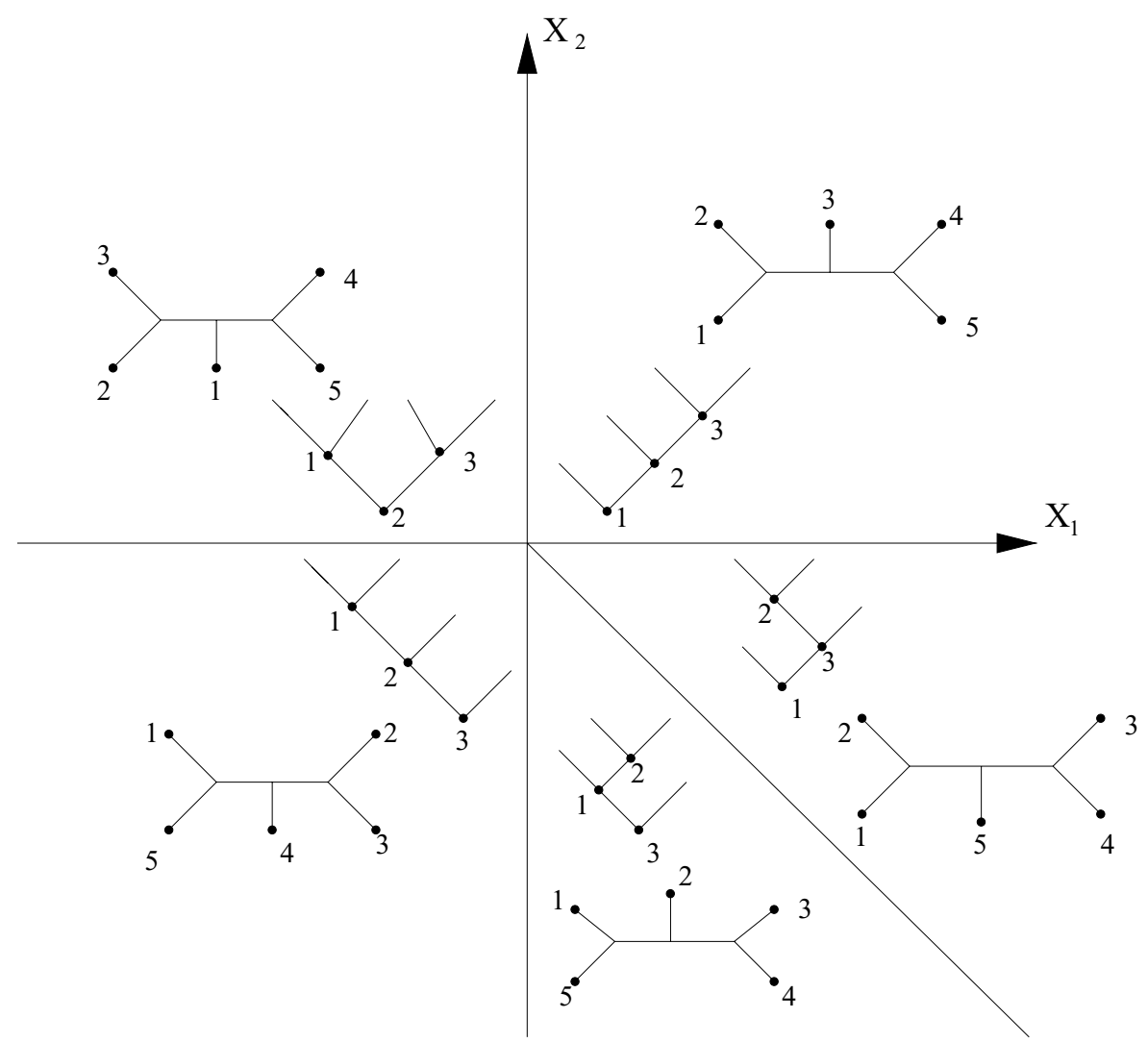

Figure 6. The fan of $\operatorname{Trop}^{+} \mathrm{Gr}_{2,5}$.

the pair $(i, j)$ the inequality

$$
x_{i}+\cdots+x_{j-1} \geq 0,
$$

while if $i>j$ then associate with $(i, j)$ the inequality

$$
x_{i}+\cdots+x_{j-1} \leq 0 \text {. }
$$

These $n-3$ inequalities define a simplicial cone $C_{T}$ in $\mathbb{R}^{n-3}$.

The result proved in [19] is the following.

Theorem 5.1 ( [19]) The $c_{n-2}$ cones $C_{T}$, as $T$ ranges over all plane binary trees with $n-1$ leaves, form the chambers of a complete fan in $\mathbb{R}^{n-3}$. Moreover, the face poset of $F_{n-3}$, with a top element $\hat{1}$ adjoined, is dual to the face lattice of the associahedron which parameterizes triangulations of the convex n-gon. 
The key step in proving that our fan $F_{2, n}$ is equal to the Stanley-Pitman fan $F_{n-3}$ is the following lemma, also proved in [19].

Lemma $5.2([19]) \quad$ Let $D_{i}=\left\{\left(x_{1}, \ldots, x_{n-3}\right) \in \mathbb{R}^{n-3}: x_{1}+\cdots+x_{i-1}=\min \left(0, x_{1}, x_{1}+\right.\right.$ $\left.\left.x_{2}, \ldots, x_{1}+\cdots+x_{n-3}\right)\right\}$. Let $\mathcal{T}_{i}$ consist of all plane binary trees with $n-1$ leaves and root i. Then $D_{i}=\cup_{T \in \mathcal{T}_{i}} C_{T}$.

Proposition 5.3 The fan $F_{2, n}$ is equal to the fan $F_{n-3}$.

Proof: First let us describe the fan $F_{2, n}$ as explicitly as possible. Note that if we label the regions of $\mathrm{Web}_{2, n}$ with the variables $x_{1}, \ldots, x_{n-3}$ from right to left, then all of the maps $\operatorname{Trop} P_{K}$ are of the form

$$
\min \left(x_{1}+x_{2}+\cdots+x_{i}, x_{1}+x_{2}+\cdots+x_{i+1}, \ldots, x_{1}+x_{2}+\cdots+x_{j}\right),
$$

where $0 \leq i \leq j \leq n$. Since this map has the same domains of linearity as the map

$$
\theta_{i j}:=\min \left(x_{i}, x_{i}+x_{i+1}, \ldots, x_{i}+\cdots+x_{j}\right)
$$

we can work with the maps $\theta_{i j}$ instead. Let $F(i, j)$ be the fan whose cones are the domains of linearity of $\theta_{i j}$. Then $F_{2, n}$ is the simultaneous refinement of all fans $F(i, j)$ where $0 \leq i \leq j \leq n$.

Now note that the previous lemma actually gives us an algorithm for determining which cone $C_{T}$ a generic point $\left(x_{1}, \ldots, x_{n-3}\right) \in \mathbb{R}^{n-3}$ lies in. Namely, if we are given such a point, compute the partial sums of $x_{1}+\cdots+x_{i-1}$, for $1 \leq i \leq n-2$. Choose $i$ such that $x_{1}+\cdots+x_{i-1}$ is the minimum of these sums. (If $i=1$, the sum is 0 .) Then the root of the tree $T$ is $i$. The left subtree of $T$ consists of vertices $\{1, \ldots, i-1\}$, and the right subtree of $T$ consists of vertices $\{i+1, \ldots, n-2\}$. We now compute $\min \left\{0, x_{1}, x_{1}+x_{2}, \ldots, x_{1}+\right.$ $\left.\cdots+x_{i-2}\right\}$ and $\min \left\{x_{i}, x_{i}+x_{i+1}, \ldots, x_{i}+\cdots+x_{n-3}\right\}$ in order to compute the roots of these two subtrees and so forth.

Now take a point $\left(x_{1}, \ldots, x_{n-3}\right)$ in a cone $C$ of $F_{2, n}$. This means that the point is in a domain of linearity for all of the piecewise linear functions $\theta_{i j}=\min \left\{x_{i}, x_{i}+x_{i+1}, \ldots, x_{i}+\right.$ $\left.\cdots+x_{j}\right\}$ where $0 \leq i \leq j \leq n$, and we take $x_{0}$ to be 0 . In other words, for each $i$ and $j$, there is a unique $k$ such that $x_{i}+\cdots+x_{k}=\min \left\{x_{i}, x_{i}+x_{i+1}, \ldots, x_{i}+\cdots+x_{j}\right\}$. In particular, we can reconstruct the tree $T$ such that $\left(x_{1}, \ldots, x_{n-3}\right) \in C_{T}$, and every point $x \in C$ belongs to this same cone $C_{T}$.

Finally, we can show by induction that $C_{T} \subset C$. (We need to show that all of the functions $\theta_{i j}$ are actually linear on $C_{T}$.) This shows that each cone $C$ in $F_{2, n}$ is actually equal to a cone $C_{T}$ in $F_{n-3}$, and conversely.

\section{Trop ${ }^{+} G r_{3,6}$ and the type $D_{4}$ associahedron}

In connection with their work on cluster algebras, Fomin and Zelevinsky [5] recently introduced certain polytopes called generalized associahedra corresponding to each Dynkin 
Table 1. Rays and Inequalities for $F_{3,6}$.

\begin{tabular}{ll}
\hline$e_{1}$ & $x_{1} \leq 5$ \\
$e_{2}$ & $x_{2} \leq 7$ \\
$e_{3}$ & $x_{3} \leq 7$ \\
$e_{4}$ & $x_{4} \leq 10$ \\
$-e_{1}$ & $-x_{1} \leq 0$ \\
$-e_{2}$ & $-x_{2} \leq-2$ \\
$-e_{3}$ & $-x_{3} \leq-2$ \\
$-e_{4}$ & $-x_{4} \leq-5$ \\
$e_{1}-e_{2}$ & $x_{1}-x_{2} \leq 0$ \\
$e_{1}-e_{3}$ & $x_{1}-x_{3} \leq 0$ \\
$e_{1}-e_{4}$ & $x_{1}-x_{4} \leq-1$ \\
$-e_{1}+e_{4}$ & $-x_{1}+x_{4} \leq 9$ \\
$e_{2}-e_{4}$ & $x_{2}-x_{4} \leq 0$ \\
$e_{3}-e_{4}$ & $x_{3}-x_{4} \leq 0$ \\
$e_{1}-e_{2}-e_{3}$ & $x_{1}-x_{2}-x_{3} \leq-3$ \\
$e_{2}+e_{3}-e_{4}$ & $x_{2}+x_{3}-x_{4} \leq 6$ \\
\hline
\end{tabular}

type, of which the usual associahedron is the type $A$ example. When we computed $F_{3,6}$, the fan associated with $\operatorname{Trop}^{+} \mathrm{Gr}_{3,6}$, we found that it was closely related to the normal fan of the type $D_{4}$ associahedron, in a way which we will now make precise. (We defer the explanation of our computations to the end of this section.)

Proposition 6.1 The $f$-vector of $F_{3,6}$ is $(16,66,98,48)$. The rays of $F_{3,6}$ are listed in Table 1, along with the inequalities defining the polytope that $F_{3,6}$ is normal to.

Using the formulas of [5], we calculated the $f$-vector of the normal fan to the type $D_{4}$ associahedron: it is $(16,66,100,50)$. More specifically, our fan has two cones which are of the form of a cone over a bipyramid. (Type FFFGG in the language of [18].) If we subdivide these two bipyramids into two tetrahedra each, then we get precisely the $D_{4}$ associahedron.

In Section 8, we will give some background on cluster algebras and formulate a conjecture which explains the relation of $F_{3,6}$ to the normal fan to the type $D_{4}$ associahedron.

We depict the intersection of $F_{3,6}$ with a sphere in figures 7 and 8 . Each of the figures is homeomorphic to a solid torus, and the two figures glue together to form the sphere $S^{3}$. The bipyramids in question have vertices $\left\{e_{2}+e_{3}-e_{4},-e_{1}, e_{2}, e_{3},-e_{1}+e_{4}\right\}$ and $\left\{e_{1}-e_{2}-e_{3},-e_{4}, e_{1}-e_{2}, e_{1}-e_{3}, e_{1}-e_{4}\right\}$.

Now we will explain how we computed $F_{3,6}$. We used two methods: the first method was to use computer software (we used both cdd + and Polymake) to compute the fan which we described in Section 4. The second method was to figure out which subfan of Trop $G r_{3,6}$ (which was explicitly described in [18]) was positive. 


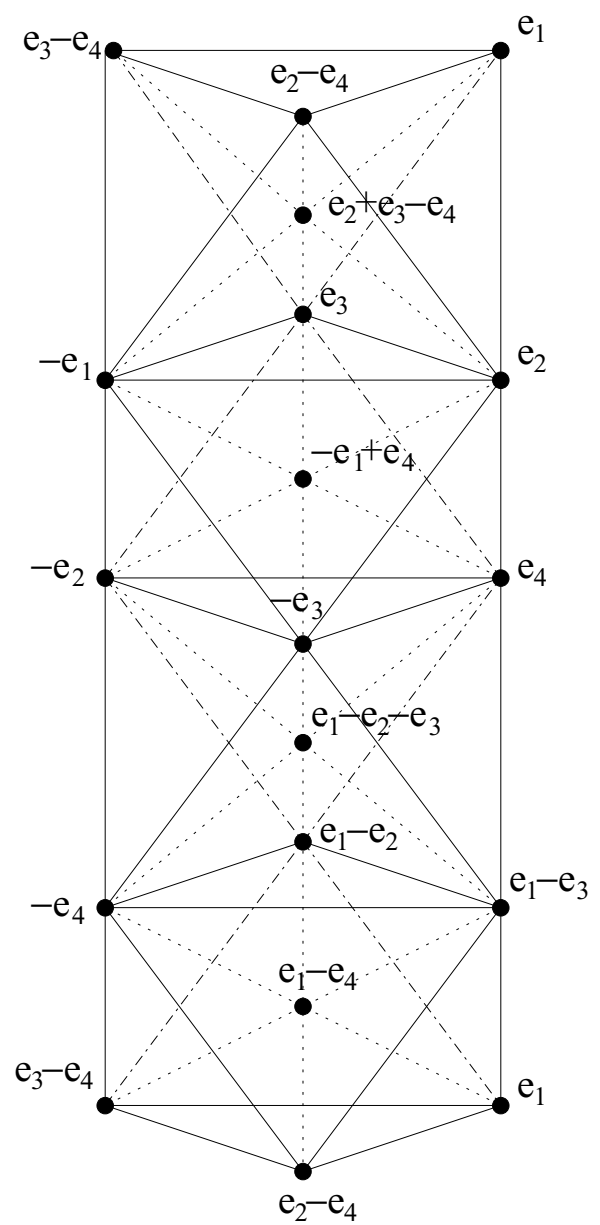

Figure 7. Glue bottom and top together to form a torus.

To implement our first method, we used the well-known result that if $F_{1}$ and $F_{2}$ are fans which are normal to polytopes $Q_{1}$ and $Q_{2}$, then the fan which is the refinement of $F_{1}$ and $F_{2}$ is normal to the Minkowski sum of $Q_{1}$ and $Q_{2}$. Since the fan $F_{k, n}$ is the simultaneous refinement of all the fans $F\left(P_{K}\right)$, we found explicit coordinates for polytopes $Q\left(P_{K}\right)$ whose normal fans were the fans $F\left(P_{K}\right)$, and had the programs cdd + and Polymake compute the Minkowski sum $Q_{k, n}$ of all of these polytopes. We then got explicit coordinates for the fan which was normal to the resulting polytope.

For the second method, we used the results in [18]: we checked which of the rays of Trop $G r_{3,6}$ did not lie $\operatorname{Trop}^{+} G r_{3,6}$, and checked which facets of Trop $G r_{3,6}$ did lie in Trop $^{+} G r_{3,6}$. As Trop ${ }^{+} G r_{3,6}$ is a closed subfan of Trop $G r_{3,6}$, this implied that every face of Trop $G r_{3,6}$ which lay in a totally positive facet was in $\operatorname{Trop}^{+} G r_{3,6}$ and every face of 


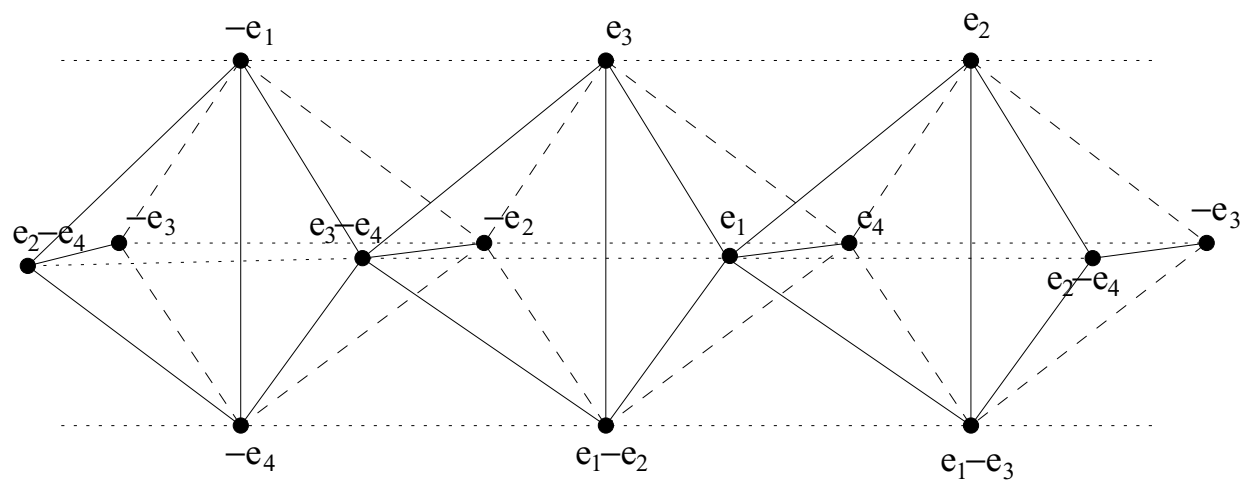

Figure 8. Glue left and right ends together to form a torus.

Trop $\mathrm{Gr}_{3,6}$ which contained a non-totally positive ray was not in $\operatorname{Trop}^{+} G r_{3,6}$; for every face of Trop $G r_{3,6}$, this proved sufficient to determine whether it was in $\operatorname{Trop}^{+}{ }^{+} r_{3,6}$ or not.

\section{7. $\operatorname{Trop}^{+} G r_{3,7}$ and the type $E_{6}$ associahedron}

As in the case of $F_{3,6}$, we used computer software to compute $F_{3,7}$, the fan associated to $\operatorname{Trop}^{+} G r_{3,7}$.

Proposition 7.1 The $f$-vector of $F_{3,7}$ is $(42,392,1463,2583,2163,693)$. Its rays are listed in Table 2, along with the inequalities defining the polytope that $F_{3,7}$ is normal to. Of the facets of this fan, 595 are simplicial, 63 have 7 vertices, 28 have 8 vertices and 7 have 9 vertices. All faces not of maximal dimension are simplicial.

Using the formulas of [5], we calculated the $f$-vector of the fan normal to the type $E_{6}$ associahedron: it is $(42,399,1547,2856,2499,833)$.

In Section 8 , we will explain why $F_{3,7}$ differs from the $E_{6}$ fan, and how one can refine $F_{3,7}$ to get a fan combinatorially equivalent to the fan dual to the type $E_{6}$ associahedron. In this refinement, the simplicial facets remain facets. The 7, 8 and 9 vertex facets split into 2 , 3 and 4 simplices respectively. The following table shows how the vertices of the 7, 8 , and 9 vertex facets are grouped into simplices.

$$
\begin{aligned}
<\mathrm{ABCDEFG}>\Longrightarrow & <\mathrm{ABCDEF}>\cup<\mathrm{ABCDEG}>\cup<\mathrm{ABCDGH}> \\
<\mathrm{ABCDEFGH}>\Longrightarrow & <\mathrm{ABCDEF}>\cup<\mathrm{ABCDFG}> \\
<\mathrm{ABCDEFGHI}>\Longrightarrow & <\mathrm{ABCDEF}>\cup<\mathrm{ABCEFG}>\cup<\mathrm{ABCFGH}> \\
& \cup<\mathrm{ABCGHI}>
\end{aligned}
$$


Table 2. Rays and Inequalities for $F_{3,7}$.

\begin{tabular}{|c|c|}
\hline$e_{1}$ & $x_{1} \leq 10$ \\
\hline$e_{2}$ & $x_{2} \leq 16$ \\
\hline$e_{3}$ & $x_{3} \leq 19$ \\
\hline$e_{4}$ & $x_{4} \leq 14$ \\
\hline$e_{5}$ & $x_{5} \leq 26$ \\
\hline$e_{6}$ & $x_{6} \leq 35$ \\
\hline$-e_{1}$ & $-x_{1} \leq-1$ \\
\hline$-e_{2}$ & $-x_{2} \leq-4$ \\
\hline$-e_{3}$ & $-x_{3} \leq-10$ \\
\hline$-e_{4}$ & $-x_{4} \leq-5$ \\
\hline$-e_{5}$ & $-x_{5} \leq-17$ \\
\hline$-e_{6}$ & $-x_{6} \leq-26$ \\
\hline$e_{1}-e_{2}$ & $x_{1}-x_{2} \leq-1$ \\
\hline$e_{1}-e_{3}$ & $x_{1}-x_{3} \leq-4$ \\
\hline$e_{1}-e_{4}$ & $x_{1}-x_{4} \leq-1$ \\
\hline$e_{1}-e_{5}$ & $x_{1}-x_{5} \leq-7$ \\
\hline$e_{1}-e_{6}$ & $x_{1}-x_{6} \leq-17$ \\
\hline$e_{2}-e_{3}$ & $x_{2}-x_{3} \leq-1$ \\
\hline$e_{2}-e_{5}$ & $x_{2}-x_{5} \leq-4$ \\
\hline$e_{2}-e_{6}$ & $x_{2}-x_{6} \leq-12$ \\
\hline$e_{3}-e_{6}$ & $x_{3}-x_{6} \leq-10$ \\
\hline$e_{4}-e_{5}$ & $x_{4}-x_{5} \leq-5$ \\
\hline$e_{4}-e_{6}$ & $x_{4}-x_{6} \leq-14$ \\
\hline$e_{5}-e_{6}$ & $x_{5}-x_{6} \leq-5$ \\
\hline$-e_{1}+e_{5}$ & $-x_{1}+x_{5} \leq 23$ \\
\hline$-e_{2}+e_{5}$ & $-x_{2}+x_{5} \leq 21$ \\
\hline$-e_{2}+e_{6}$ & $-x_{2}+x_{6} \leq 28$ \\
\hline$e_{2}+e_{4}-e_{5}$ & $x_{2}+x_{4}-x_{5} \leq 8$ \\
\hline$e_{2}+e_{4}-e_{6}$ & $x_{2}+x_{4}-x_{6} \leq 1$ \\
\hline$e_{3}+e_{4}-e_{6}$ & $x_{3}+x_{4}-x_{6} \leq 3$ \\
\hline$e_{3}+e_{5}-e_{6}$ & $x_{3}+x_{5}-x_{6} \leq 13$ \\
\hline$e_{1}-e_{2}+e_{6}$ & $x_{1}-x_{2}+x_{6} \leq 33$ \\
\hline$e_{1}-e_{2}-e_{4}$ & $x_{1}-x_{2}-x_{4} \leq-7$ \\
\hline$e_{1}-e_{3}-e_{4}$ & $x_{1}-x_{3}-x_{4} \leq-12$ \\
\hline$e_{1}-e_{3}-e_{5}$ & $x_{1}-x_{3}-x_{5} \leq-19$ \\
\hline$e_{2}-e_{3}-e_{5}$ & $x_{2}-x_{3}-x_{5} \leq-17$ \\
\hline$-e_{1}+e_{5}-e_{6}$ & $-x_{1}+x_{5}-x_{6} \leq-7$ \\
\hline$e_{1}+e_{2}-e_{3}-e_{5}$ & $x_{1}+x_{2}-x_{3}-x_{5} \leq-9$ \\
\hline$e_{2}+e_{4}-e_{5}-e_{6}$ & $x_{2}+x_{4}-x_{5}-x_{6} \leq-19$ \\
\hline$e_{1}-e_{2}-e_{4}+e_{6}$ & $x_{1}-x_{2}-x_{4}+x_{6} \leq 26$ \\
\hline$e_{2}-e_{3}+e_{4}-e_{5}$ & $x_{2}-x_{3}+x_{4}-x_{5} \leq-7$ \\
\hline$-e_{1}+e_{3}+e_{5}-e_{6}$ & $-x_{1}+x_{3}+x_{5}-x_{6} \leq 11$ \\
\hline
\end{tabular}




\section{Cluster algebras}

Cluster algebras are commutative algebras endowed with a certain combinatorial structure, introduced in [2] and expected to be relevant in studying total positivity and homogeneous spaces, such as Grassmannians.

We will not attempt to give a precise definition of a cluster algebra here, but will rather describe their key properties. Slightly varying definitions can be found in [2, 3] and [17]; we follow [17] but do not believe these small variations are important.

A cluster algebra is an algebra $\mathcal{A}$ over a field $k$, which in our examples can be thought of as $\mathbb{R}$. Additionally, a cluster algebra carries two subsets $C$ and $X \subset \mathcal{A}$, known as the coefficient variables and the cluster variables. $C$ is finite, but $X$ may be finite or infinite. If $X$ is finite, $\mathcal{A}$ is known as a cluster algebra of finite type. There is also a nonnegative integer $r$ associated to a cluster algebra and known as the rank of the algebra.

There is a pure $(r-1)$-dimensional simplicial complex called the cluster complex whose vertices are the elements of $X$ and whose maximal simplices are called clusters. We will denote the cluster complex by $S(\mathcal{A})$. If $x \in X$ and $\Delta \in S(\mathcal{A})$ is a cluster containing $x$, there is always a unique cluster $\Delta^{\prime}$ with $\Delta \cap \Delta^{\prime}=\Delta \backslash\{x\}$. Let $\Delta^{\prime}=(\Delta \backslash\{x\}) \cup\left\{x^{\prime}\right\}$. Then there is a relation $x x^{\prime}=B$ where $B$ is a binomial in the variables of $\left(\Delta \cap \Delta^{\prime}\right) \cup C$.

For any $x \in X$ and any cluster $\Delta, x$ is a subtraction-free rational expression in the members of $\Delta \cup C$ and is also a Laurent polynomial in the members of $\Delta \cup C$. Conjecturally, this Laurent polynomial has non-negative coefficients. Note that this conjecture does not follow from the preceding sentence: $\frac{x^{3}+y^{3}}{x+y}=x^{2}-x y+y^{2}$ is a subtraction-free expression in $x$ and $y$, and a polynomial in $x$ and $y$, but it is not a polynomial with positive coefficients.

It was demonstrated in [17] that the coordinate rings of Grassmannians have natural cluster algebra structures. Usually these cluster algebras are of infinite type, making them hard to work with in practice, but in the cases of $G r_{2, n}, G r_{3, k}$ for $k \leq 8$ and their duals, we get cluster algebras of finite type.

In the case of $G r_{2, n}$, the coefficient set $C$ is $\left\{\Delta_{12}, \Delta_{23}, \ldots, \Delta_{(n-1) n}, \Delta_{1 n}\right\}$ and the set of cluster variables $X$ is $\left\{\Delta_{i j}: i<j\right.$ and $\left.i-j \not \equiv \pm 1 \bmod n\right\}$. (Note that these $\Delta$ 's are Plücker coordinates and not simplices.) Label the vertices of an $n$-gon in clockwise order with the indices $\{1,2, \ldots, n\}$ and associate to each member of $X \cup C$ the corresponding chord of the $n$-gon. The clusters of $G r_{2, n}$ correspond to the collections of chords which triangulate the $n$-gon. Thus, $S(\mathcal{A})$ in this example is (as an abstract simplicial complex) isomorphic to the dual of the associahedron. Since we have shown that the fan of $\operatorname{Trop}^{+} G r_{2, n}$ is combinatorially equivalent to the normal fan of the associahedron, it follows that $\operatorname{Trop}^{+} G r_{2, n}$ is (combinatorially) the cone on $S(\mathcal{A})$.

In the case of $G r_{3,6}$, the coefficient set $C$ is equal to $\left\{\Delta_{123}, \Delta_{234}, \Delta_{345}, \Delta_{456}, \Delta_{561}, \Delta_{612}\right\}$. $X$ contains the other 14 Plücker coordinates, but it also contains two unexpected elements: $\Delta_{134} \Delta_{256}-\Delta_{156} \Delta_{234}$ and $\Delta_{236} \Delta_{145}-\Delta_{234} \Delta_{156}$. By definition, all Plücker coordinates are positive on the totally positive Grassmannian, so by the results above on subtraction-free rational expressions, these new coordinates are positive on the totally positive Grassmannian as well.

The new coordinates turn out to be Laurent polynomials with positive coefficients in the region variables of Section 3. Thus, we can tropicalize these Laurent polynomials and 
associate a fan to each of them. When we refine $F_{3,6}$ by these fans, the refinement subdivides the two bipyramids and yields precisely the normal fan to the $D_{4}$ associahedron, which is again the cone over $S(\mathcal{A})$.

In the case of $G r_{3,7}, C$ again consists of $\left\{\Delta_{i(i+1)(i+2)}\right\}$ where indices are modulo 7. $X$ contains all of the other Plücker variables and the pullbacks to $G r_{3,7}$ of the two new cluster variables of $G r_{3,6}$, along with the 7 rational coordinate projections $G r_{3,7} \rightarrow G r_{3,6}$. Thus, $X$ contains 28 Plücker variables and 14 other variables.

As in the case of $G r_{3,6}$, the 14 new variables are Laurent polynomials with positive coefficients in the region variables of Section 3, so to each one we can associate a corresponding fan. When we refine $F_{3,7}$ by these 14 new fans, we get a fan combinatorially equivalent to the fan normal to the $E_{6}$ associahedron.

We can describe what we have seen in each of these Grassmannian examples in terms of the general language of cluster algebras as follows:

Observation when $\mathcal{A}$ is the coordinate ring of a Grassmannian. Embed $\operatorname{Spec} \mathcal{A}$ in affine space by the variables $X \sqcup C$. Then $\operatorname{Trop}^{+} \operatorname{Spec} \mathcal{A}$ is a fan with lineality space of dimension $|C|$. After taking the quotient by this lineality space, we get a simplical fan abstractly isomorphic to the cone over $S(\mathcal{A})$.

This observation does not quite hold for an arbitrary cluster algebra of finite type. For example, if we take the cluster algebra of $G r_{2,6}$ and set all coefficient variables equal to 1 , we get a different cluster algebra which is still of type $A_{3}$. However, when we compute the positive part of the corresponding tropical variety, we get a fan whose lineality space has dimension 1 , not 0 as the above would predict. Our fan is a cone over a hexagon cross a 1-dimensional lineality space, which is a coarsening of the fan normal to the type $A_{3}$ associahedron. Based on this and other small examples, it seems that in order to see the entire cone over $S(\mathcal{A})$, one needs to use "enough" coefficients.

Conjecture 8.1 Let $\mathcal{A}$ be a cluster algebra of finite type over $\mathbb{R}$ and $S(\mathcal{A})$ its associated cluster complex. If the lineality space of $\operatorname{Trop}^{+} \operatorname{Spec} \mathcal{A}$ has dimension $|C|$ then $\operatorname{Trop}^{+} \operatorname{Spec} \mathcal{A}$ modulo its lineality space is a simplicial fan abstractly isomorphic to the cone over $S(\mathcal{A})$. If the condition on the lineality space does not hold, the resulting fan is a coarsening of the cone over $S(\mathcal{A})$.

Remark The condition on the lineality space can be restated without mentioning tropicalizations. Consider the torus $\left(\mathbb{R}^{*}\right)^{X \cup C}$ acting on the affine space $\mathbb{R}^{X \cup C}$ and let $G$ be the subgroup taking $\operatorname{Spec} \mathcal{A}$ to itself. We want to require that $\operatorname{dim} G=|C|$.

Remark In the notation of [2] and [3], the condition on the dimension of the lineality space is equivalent to requiring that the matrix $\tilde{B}$ be of full rank. We thank Andrei Zelevinsky for pointing this out to us.

Note how surprising this conjecture is in light of how the two complexes are computed. The fan described in the conjecture is computed as the refinement of a number of fans, indexed by the vertices of $S(\mathcal{A})$. That the rays of this fan, which arise as the intersections 
of many hypersurfaces, should again be in bijection with the vertices of $S(\mathcal{A})$ is quite unexpected.

We expect an analogous statement to hold for infinite type cluster algebras.

\section{Acknowledgments}

We are grateful to Bernd Sturmfels for suggesting this problem to us, and for advice throughout our work. In addition, we thank Komei Fukuda and Michael Joswig for their help in performing the polyhedral computations and Joshua Scott, Richard Stanley, and Andrei Zelevinsky for their useful comments.

\section{References}

1. F. Chapoton, S. Fomin, and A. Zelevinsky, "Polytopal realizations of generalized associahedra," Canadian Mathematical Bulletin 45 (2002), 537-566.

2. S. Fomin and A. Zelevinsky, "Cluster algebras I: Foundations," Journal of the AMS 15 (2002), 497529.

3. S. Fomin and A. Zelevinsky, "Cluster algebras II: Finite type classification," Inventiones Mathematicae 154 (2003), 63-121.

4. S. Fomin and A. Zelevinsky, "Total positivity: Tests and parameterizations," The Mathematical Intelligencer 22 (2000), 23-33.

5. S. Fomin and A. Zelevinsky, "Y-systems and generalized associahedra," Annals of Mathematics 158(3) (2003), 977-1018.

6. K. Fukuda, CDD-A C-implementation of the double description method, available from http://www . cs.mcgill.ca/ fukuda/soft/cdd_home/cdd.html.

7. E. Gawrilow and M. Joswig, polymake, available from http://www . math.tu-berlin.de/polymake/.

8. I. Gessel and G. Viennot, "Binomial determinants, paths and hook length formulae," Adv. In Math. 58 (1985), $300-321$.

9. D. Handelman, "Positive polynomials and product type actions of compact groups," Mem. Amer. Math. Soc. 320 (1985).

10. G. Lusztig, "Introduction to total positivity," in "Positivity in Lie theory: Open problems," (Ed.) J. Hilgert, J.D. Lawson, K.H. Neeb and E.B. Vinberg (Eds.), de Gruyter Berlin, 1998, pp. 133-145.

11. G. Lusztig, "Total positivity in partial flag manifolds," Representation Theory, 2 (1998), 70-78.

12. G. Lusztig, "Total positivity in reductive groups," in Lie theory and geometry: In honor of Bertram Kostant, Progress in Mathematics 123, Birkhauser, 1994, 531-568.

13. M. Einsiedler and S. Tuncel, "When does a polynomial ideal contain a positive polynomial?," in Effective Methods in Algebraic Geometry, J. Pure Appl. Algebra 164(1/2) (2001), 149-152.

14. G. Mikhalkin, "Counting curves via lattice paths in polygons," C. R. Math. Acad. Sci. Paris 336 (2003), 629-634.

15. A. Postnikov, "Webs in totally positive Grassman cells," in preparation.

16. J. Richter-Gebert, B. Sturmfels, and T. Theobald, "First steps in tropical geometry," 29 pages, http: //www.arxiv.org/math.AG/0306366, to appear in "Idempotent Mathematics and Mathematical Physics", (eds. G. Litvinov and V. Maslov), Proceedings Vienna, 2003.

17. J. Scott, "Grassmannians and cluster algebras," preprint, http : //www.arxiv.org/math.CO/0311148

18. D. Speyer and B. Sturmfels, "The tropical Grassmannian," Adv. Geom. 4 (2004), 389-411.

19. R. Stanley and J. Pitman, "A polytope related to empirical distribution, plane trees, parking functions, and the associahedron," Discrete Comput. Geometry 27 (2002), 603-634. 
20. B. Sturmfels, "Gröbner bases and convex polytopes," American Mathematical Society, Providence, 1991.

21. B. Sturmfels, "Solving systems of polynomial equations," American Mathematical Society, Providence, 2002.

22. L. Williams, "Enumeration of totally positive Grassmann cells," Adv. Math. 190 (2005), 319-342. 